TAIWANESE JOURNAL OF MATHEMATICS

Vol. 15, No. 4, pp. 1527-1543, August 2011

This paper is available online at http://www.tjm.nsysu.edu.tw/

\title{
ON THE BOUNDEDNESS OF MULTILINEAR OPERATORS ON WEIGHTED HERZ-MORREY SPACES
}

\author{
Xiangxing Tao* and Huihui Zhang
}

\begin{abstract}
Boundedness of multilinear operators on weighted Herz-Morrey spaces is established in this paper. The weak estimates on endpoints are also derived. As a special case, the conclusions can apply to multilinear CalderonZygmund operators.
\end{abstract}

\section{INTRODUCTION}

In recent years, the study of multilinear integrals has been received more attention, which is motivated not only as the generalization of the linear theory but also the natural appearance of multilinear singular integral theory. In 2002, Grafakos and Torres [2] studied the boundedness of multilinear Calder on-Zygmund operators on the product of Lebesgue spaces and the endpoint weak estimates; and they extended it to a weighted version in [3]. Recently, the authors have studied the boundedness of the multilinear fractional integrals on Herz-Morrey spaces in [6] [7] and [10]. In this paper, we focus on the boundedness of the multilinear singular integral operators on weighted Herz-Morrey spaces and their weak estimates on endpoints. As a special case, our conclusions can apply to multilinear Calderon-Zygmund operators and thus extend the related theorems appeared in [2] and [3], and also include the related results in [9] and [11] in which the boundedness on weighted Herz spaces were considered.

In fact, we consider the following general multilinear operator

(1.1) $\mathcal{T}\left(f_{1}, \ldots, f_{m}\right)(x)=\int_{\left(\mathbb{R}^{n}\right)^{m}} \mathcal{K}\left(x, y_{1}, \ldots, y_{m}\right) f_{1}\left(y_{1}\right) \ldots f_{m}\left(y_{m}\right) d y_{1} \ldots d y_{m}$

Received September 12, 2009, accepted February 27, 2010.

Communicated by Chin-Cheng Lin.

2000 Mathematics Subject Classification: 42B20, 42B35.

Key words and phrases: Multilinear operator, Caderon-Zygmund, Weighted Herz-Morrey space, Weak Herz-Morrey space.

This work was supported partly by National Natural Science Foundation of China under grant \#10771110 and \#10471069, and founded by New Century 151 Talent Project of Zhejiang Province.

*Corresponding author. 
with the kernel $\mathcal{K}$ satisfying the size condition

$$
\left|\mathcal{K}\left(x, y_{1}, \ldots, y_{m}\right)\right| \leq C\left(\sum_{i=1}^{m}\left|x-y_{i}\right|\right)^{-m n} .
$$

This class of multilinear operators include the $m$-linear Calderon-Zygmund operators, which are associated with an $m$-linear Calderon-Zygmund kerneal $\mathcal{K}$ defined away from the diagonal $y_{0}=y_{1}=\ldots=y_{m}$ in $\left(\mathbb{R}^{n}\right)^{m+1}$ satisfying

$$
\left|\mathcal{K}\left(y_{0}, y_{1}, \ldots, y_{m}\right)\right| \leq C\left(\sum_{i, j=0}^{m}\left|y_{i}-y_{j}\right|\right)^{-m n}
$$

and

$$
\left|\nabla \mathcal{K}\left(y_{0}, y_{1}, \ldots, y_{m}\right)\right| \leq C\left(\sum_{i, j=0}^{m}\left|y_{i}-y_{j}\right|\right)^{-(m n+1)} .
$$

Definition 1.1. A non-negative function $u$ is said to be a Muckenhoupt $A_{p}$ weight, denote by $u \in A_{p}$, if for any ball $B$ on $\mathbb{R}^{n}$, there exists a constant $C>0$ independent of the ball $B$ such that

$$
\begin{array}{cl}
\frac{1}{|B|} \int_{B} u(x) d x\left(\frac{1}{|B|} \int_{B} u(x)^{-\frac{1}{p-1}} d x\right)^{p-1} \leq C, & \text { when } 1<p<\infty \\
\frac{1}{|B|} \int_{B} u(x) d x \leq C \inf _{x \in B} u(x), & \text { when } p=1 .
\end{array}
$$

It's well known that $A_{p} \subset A_{\infty}$ for any $1 \leq p<\infty$, thus for any $u \in A_{p}$ there exists $0<\delta_{u} \leq p$ such that

$$
C_{1}\left(\frac{\left|B_{1}\right|}{\left|B_{2}\right|}\right)^{p} \leq \frac{u\left(B_{1}\right)}{u\left(B_{2}\right)} \leq C_{2}\left(\frac{\left|B_{1}\right|}{\left|B_{2}\right|}\right)^{\delta_{u}} \quad \text { for any } B_{1} \subset B_{2} .
$$

From the works of Grafakos and Torres in [2] and [3], we have

Theorem 1.2. Let $1<q_{1}, \ldots, q_{m}, q<\infty$ and $\frac{1}{q}=\frac{1}{q_{1}}+\ldots+\frac{1}{q_{m}}$. Then the m-linear Calderon-Zygmund operator $\mathcal{T}\left(f_{1}, \ldots, f_{m}\right)$ is bounded from $L^{q_{1}}\left(\mathbb{R}^{n}\right) \times$ $\ldots \times L^{q_{m}}\left(\mathbb{R}^{n}\right)$ to $L^{q}\left(\mathbb{R}^{n}\right)$.

Moreover, if $u_{i} \in A_{q_{i}}\left(\mathbb{R}^{n}\right), i=1,2, \ldots, m$ and $u^{\frac{1}{q}}=u_{1}^{\frac{1}{q_{1}}} u_{2}^{\frac{1}{q_{2}}} \ldots u_{m}^{\frac{1}{q_{m}}}$, then

$$
\left\|\mathcal{T}\left(f_{1}, \ldots, f_{m}\right)\right\|_{q, u} \leq C \prod_{i=1}^{m}\left\|f_{i}\right\|_{q_{i}, u_{i}},
$$

where we denote by $\|f\|_{q, u}=\|f\|_{L_{u}^{q}}=\left(\int_{\mathbb{R}^{n}}|f(x)|^{q} u(x) d x\right)^{\frac{1}{q}}$. 
In order to state our theorems, we first introduce the weighted Herz-Morrey spaces, which are the generalization of the classical Morrey space and Herz space, date back to Beurling [1] and Herz [4]. One may see [5] for the weighted Herz spaces. Throughout the whole paper, let $B_{k}=\left\{x \in \mathbb{R}^{n}:|x|<2^{k}\right\}, E_{k}=$ $B_{k} \backslash B_{k-1}$ and $\chi_{k}=\chi_{E_{k}}$.

Definition 1.3. Suppose $\alpha \in R, 0 \leq q<\infty, 0 \leq p<\infty, 0 \leq \lambda<\infty$, and $v, u$ are two nonnegative weights on $\mathbb{R}^{n}$, then the homogeneous weighted Herz-Morrey space $M \dot{K}_{p, q}^{\alpha, \lambda}(v, u)$ is defined by

$$
M \dot{K}_{p, q}^{\alpha, \lambda}(v, u)=\left\{f \in L_{l o c}^{q}\left(\mathbb{R}^{n} \backslash\{0\}, u\right):\|f\|_{M \dot{K}_{p, q}^{\alpha, \lambda}(v, u)}<\infty\right\}
$$

with the norm

$$
\|f\|_{M \dot{K}_{p, q}^{\alpha, \lambda}(v, u)}=\sup _{k_{0} \in Z} v\left(B_{k_{0}}\right)^{\frac{-\lambda}{n}}\left\{\sum_{k=-\infty}^{k_{0}} v\left(B_{k}\right)^{\frac{\alpha p}{n}}\left\|f \chi_{k}\right\|_{L_{u}^{q}}^{p}\right\}^{\frac{1}{p}} .
$$

The homogeneous weighted weak Herz-Morrey space $W M \dot{K}_{p, q}^{\alpha, \lambda}(v, u)$ is defined by

$$
W M \dot{K}_{p, q}^{\alpha, \lambda}(v, u)=\left\{f \in L_{l o c}^{q}\left(\mathbb{R}^{n} \backslash\{0\}, u\right):\|f\|_{W M \dot{K}_{p, q}^{\alpha, \lambda}(v, u)}<\infty\right\}
$$

with the norm

$$
\|f\|_{W M \dot{K}_{p, q}^{\alpha, \lambda}(v, u)}=\sup _{\substack{k_{0} \in Z \\ t>0}} v\left(B_{k_{0}}\right)^{\frac{-\lambda}{n}} t\left\{\sum_{k=-\infty}^{k_{0}} v\left(B_{k}\right)^{\frac{\alpha p}{n}}\left[u\left(\left\{x \in E_{k}:|f(x)|>t\right\}\right)\right]^{\frac{p}{q}}\right\}^{\frac{1}{p}}
$$

Remark 1.4. If $v=u=1$, then $M \dot{K}_{p, q}^{\alpha, \lambda}(v, u)$ is the homogeneous Herz-Morrey space $M \dot{K}_{p, q}^{\alpha, \lambda}\left(\mathbb{R}^{n}\right), W M \dot{K}_{p, q}^{\alpha, \lambda}(v, u)$ is the homogeneous weak Herz-Morrey space $W M \dot{K}_{p, q}^{\alpha, \lambda}\left(\mathbb{R}^{n}\right)$. If $\lambda=0$, then $M \dot{K}_{p, q}^{\alpha, \lambda}(v, u)$ reduces to the weighted homogeneous Herz space $\dot{K}_{q}^{\alpha, p}(v, u), W M \dot{K}_{p, q}^{\alpha, \lambda}(v, u)$ becomes the homogeneous weighted weak Herz space $W \dot{K}_{q}^{\alpha, p}(v, u)$.

Next we always suppose that $\mathcal{T}$ is a multilinear operator defined as (1.1) with the condition (1.2); And always assume that

$$
u^{\frac{1}{q}}=u_{1}^{\frac{1}{q_{1}}} u_{2}^{\frac{1}{q_{2}}} \ldots u_{m}^{\frac{1}{q_{m}}}
$$

and

$$
\frac{1}{p}=\sum_{i=1}^{m} \frac{1}{p_{i}}, \quad \frac{1}{q}=\sum_{i=1}^{m} \frac{1}{q_{i}}, \quad \alpha=\sum_{i=1}^{m} \alpha_{i}, \quad \lambda=\sum_{i=1}^{m} \lambda_{i} .
$$

We will prove the following three theorems. 
Theorem 1.5. Let $0<p_{i}<\infty, 1<q_{i}<\infty, v \in A_{q_{v}}$ with $1 \leq q_{v}<\infty$, $u_{i} \in A_{q_{u_{i}}}$ with $1 \leq q_{u_{i}}<q_{i}$, and let $\alpha_{i} q_{v}<n\left(1-\frac{q_{u_{i}}}{q_{i}}\right), \alpha_{i} \delta_{v}>\frac{-n \delta_{u_{i}}}{q_{i}}+\lambda_{i} q_{v}$ for $i=1,2, \ldots, m$. If $\mathcal{T}$ is bounded from $L_{u_{1}}^{q_{1}}\left(\mathbb{R}^{n}\right) \times \ldots \times L_{u_{m}}^{q_{m}}\left(\mathbb{R}^{n}\right)$ to $L_{u}^{q}\left(\mathbb{R}^{n}\right)$, then

$$
\left\|\mathcal{T}\left(f_{1}, \ldots, f_{m}\right)\right\|_{M \dot{K}_{p, q}^{\alpha, \lambda}(v, u)} \leq C \prod_{i=1}^{m}\left\|f_{i}\right\|_{M \dot{K}_{p_{i}, q_{i}}^{\alpha_{i}, \lambda_{i}}\left(v, u_{i}\right)}
$$

with the constant $C$ independent of $\left(f_{1}, \ldots, f_{m}\right)$.

Theorem 1.6. Let $0<p_{i}<\infty, 1 \leq q_{i}<\infty, v \in A_{q_{v}}$ with $1 \leq q_{v}<\infty$, $u_{i} \in A_{q_{u_{i}}}$ with $1 \leq q_{u_{i}}<q_{i}$, and let $\alpha_{i} q_{v}<n\left(1-\frac{q_{u_{i}}}{q_{i}}\right), \alpha_{i} \delta_{v}>\frac{-n \delta_{u_{i}}}{q_{i}}+\lambda_{i} q_{v}$ for $i=1,2, \ldots, m$. If $\mathcal{T}$ is bounded from $L_{u_{1}}^{q_{1}}\left(\mathbb{R}^{n}\right) \times \ldots \times L_{u_{m}}^{q_{m}}\left(\mathbb{R}^{n}\right)$ to $W L_{u}^{q}\left(\mathbb{R}^{n}\right)$, then

$$
\left\|\mathcal{T}\left(f_{1}, \ldots, f_{m}\right)\right\|_{W M \dot{K}_{p, q}^{\alpha, \lambda}(v, u)} \leq C \prod_{i=1}^{m}\left\|f_{i}\right\|_{M \dot{K}_{p_{i}, q_{i}}^{\alpha_{i}, \lambda_{i}}\left(v, u_{i}\right)}
$$

with the constant $C$ independent of $\left(f_{1}, \ldots, f_{m}\right)$.

Theorem 1.7. Let $0<p_{i}<\infty, 1<q_{i}<\infty, v \in A_{q_{v}}$ with $1 \leq q_{v}<\infty$, $u_{i} \in A_{q_{u_{i}}}$ with $1 \leq q_{u_{i}}<q_{i}$, and let $\alpha_{i} q_{v} \leq n\left(1-\frac{q_{u_{i}}}{q_{i}}\right), \alpha_{i} \delta_{v}>\frac{-n \delta_{u_{i}}}{q_{i}}+\lambda_{i} \delta_{v}$ for $i=1,2, \ldots, m$. If $\mathcal{T}$ is bounded from $L_{u_{1}}^{q_{1}}\left(\mathbb{R}^{n}\right) \times \ldots \times L_{u_{m}}^{q_{m}}\left(\mathbb{R}^{n}\right)$ to $W L_{u}^{q}\left(\mathbb{R}^{n}\right)$, then

$$
\left\|\mathcal{T}\left(f_{1}, \ldots, f_{m}\right)\right\|_{W M \dot{K}_{p, q}^{\alpha, \lambda}(v, u)} \leq C \prod_{i=1}^{m}\left\|f_{i}\right\|_{M \dot{K}_{p_{i}, q_{i}}^{\alpha_{i}, \lambda_{i}}\left(v, u_{i}\right)}
$$

with the constant $C$ independent of $\left(f_{1}, \ldots, f_{m}\right)$.

Letting $v=u_{1}=u_{2}=\cdots=u_{m}=1$, we obtain the following unweighted boundedness for $\mathcal{T}$.

Corollary 1.8. Let $0<p_{i}<\infty, 1<q_{i}<\infty$, and $\frac{-n}{q_{i}}+\lambda_{i}<\alpha_{i}<n\left(1-\frac{1}{q_{i}}\right)$ for $i=1,2, \ldots, m$. If $\mathcal{T}$ is bounded from $L^{q_{1}}\left(\mathbb{R}^{n}\right) \times \ldots \times L^{q_{m}}\left(\mathbb{R}^{n}\right)$ to $L^{q}\left(\mathbb{R}^{n}\right)$, then

$$
\left\|\mathcal{T}\left(f_{1}, \ldots, f_{m}\right)\right\|_{M \dot{K}_{p, q}^{\alpha, \lambda}} \leq C \prod_{i=1}^{m}\left\|f_{i}\right\|_{M \dot{K}_{p_{i}, q_{i}}^{\alpha_{i}, \lambda_{i}}}
$$

with the constant $C$ independent of $\left(f_{1}, \ldots, f_{m}\right)$

Corollary 1.9. Let $0<p_{i}<\infty, 1 \leq q_{i}<\infty$, and $\frac{-n}{q_{i}}+\lambda_{i}<\alpha_{i}<n\left(1-\frac{1}{q_{i}}\right)$ for $i=1,2, \ldots, m$. If $\mathcal{T}$ is bounded from $L^{q_{1}}\left(\mathbb{R}^{n}\right) \times \ldots \times L^{q_{m}}\left(\mathbb{R}^{n}\right)$ to $W L^{q}\left(\mathbb{R}^{n}\right)$, then

$$
\left\|\mathcal{T}\left(f_{1}, \ldots, f_{m}\right)\right\|_{W M \dot{K}_{p, q}^{\alpha, \lambda}} \leq C \prod_{i=1}^{m}\left\|f_{i}\right\|_{M \dot{K}_{p_{i}, q_{i}}^{\alpha_{i}, \lambda_{i}}}
$$

with the constant $C$ independent of $\left(f_{1}, \ldots, f_{m}\right)$. 
Corollary 1.10. Let $0<p_{i}<\infty, 1<q_{i}<\infty$, and $\frac{-n}{q_{i}}+\lambda_{i}<\alpha_{i} \leq n\left(1-\frac{1}{q_{i}}\right)$ for $i=1,2, \ldots, m$. If $\mathcal{T}$ is bounded from $L^{q_{1}}\left(\mathbb{R}^{n}\right) \times \ldots \times L^{q_{m}}\left(\mathbb{R}^{n}\right)$ to $W L^{q}\left(\mathbb{R}^{n}\right)$, then

$$
\left\|\mathcal{T}\left(f_{1}, \ldots, f_{m}\right)\right\|_{W M \dot{K}_{p, q}^{\alpha, \lambda}} \leq C \prod_{i=1}^{m}\left\|f_{i}\right\|_{M \dot{K}_{p_{i}, q_{i}}^{\alpha_{i}, q_{i}}}
$$

with the constant $C$ independent of $\left(f_{1}, \ldots, f_{m}\right)$.

\section{The Proof of Theorem 1.5}

Without loss of generality, we only prove the theorems for the case $m=2$. We introduce some notations for convenience. For $k, i \in Z, \tau=1,2$, set

$$
E_{\tau}(k, i)= \begin{cases}2^{(k-i)\left[-n+\alpha_{\tau} q_{v}+n q_{u_{\tau}} / q_{\tau}\right]}, & \text { if } i \leq k-2 \\ 1, & \text { if } k-1 \leq i \leq k+1 . \\ 2^{(k-i)\left[\alpha_{\tau} \delta_{v}+n \delta_{u_{\tau}} / q_{\tau}\right]}, & \text { if } i \geq k+2\end{cases}
$$

Lemma 2.1. Let $v \in A_{q_{v}}$ with $1 \leq q_{v}<\infty$, and $u_{\tau} \in A_{q_{u_{\tau}}}$ with $1 \leq q_{u_{\tau}}<q_{\tau}$, $\tau=1$, 2. If $\mathcal{T}$ is bounded from $L_{u_{1}}^{q_{1}}\left(\mathbb{R}^{n}\right) \times L_{u_{2}}^{q_{2}}\left(\mathbb{R}^{n}\right)$ to $L_{u}^{q}\left(\mathbb{R}^{n}\right)$, then

$$
\begin{aligned}
& v\left(B_{k}\right)^{\alpha / n}\left\|\mathcal{T}\left(f \chi_{i}, g \chi_{j}\right) \chi_{k}\right\|_{q, u} \\
\leq & C E_{1}(k, i) v\left(B_{i}\right)^{\alpha_{1} / n}\left\|f \chi_{i}\right\|_{q_{1}, u_{1}} \times E_{2}(k, j) v\left(B_{j}\right)^{\alpha_{2} / n}\left\|g \chi_{j}\right\|_{q_{2}, u_{2}}
\end{aligned}
$$

for each $k, i, j$, where the constant $C$ is independent of $k, i, j$.

Proof. In the case $k-1 \leq i \leq k+1, k-1 \leq j \leq k+1$, we note that $v\left(B_{k}\right) \sim v\left(B_{i}\right) \sim v\left(B_{j}\right)$ because of $v \in A_{\infty}$. So by the boundedness of $\mathcal{T}$ we obtain

$$
\begin{aligned}
& v\left(B_{k}\right)^{\alpha / n}\left\|\mathcal{T}\left(f \chi_{i}, g \chi_{j}\right) \chi_{k}\right\|_{q, u} \\
\leq & C\left[v\left(B_{i}\right)\right]^{\alpha_{1} / n}\left\|f \chi_{i}\right\|_{q_{1}, u_{1}}\left[v\left(B_{j}\right)\right]^{\alpha_{2} / n}\left\|g \chi_{j}\right\|_{q_{2}, u_{2}} .
\end{aligned}
$$

In the other cases, we see that $\left|x-y_{1}\right|+\left|x-y_{2}\right| \sim 2^{\max (k, i, j)}$ for $x \in E_{k}$, $y_{1} \in E_{i}, y_{2} \in E_{j}$. Thus applying the Hölder inequality and the condition $u_{\tau} \in$ $A_{q_{u_{\tau}}}\left(\mathbb{R}^{n}\right) \subseteq A_{q_{\tau}}\left(\mathbb{R}^{n}\right)$, we have

$$
\begin{aligned}
& \left|\mathcal{T}\left(f \chi_{i}, g \chi_{j}\right) \chi_{k}(x)\right| \leq C 2^{-2 \max (k, i, j) n}\left\|f \chi_{i}\right\|_{1}\left\|g \chi_{j}\right\|_{1} \\
\leq & C 2^{-2 \max (k, i, j) n}\left\|f \chi_{i}\right\|_{q_{1}, u_{1}}\left[u_{1}^{-\frac{1}{q_{1}-1}}\left(B_{i}\right)\right]^{1 / q_{1}^{\prime}}\left\|g \chi_{j}\right\|_{q_{2}, u_{2}}\left[u_{2^{-\frac{1}{q_{2}-1}}}\left(B_{j}\right)\right]^{1 / q_{2}^{\prime}} \\
\leq & \frac{C 2^{-2 \max (k, i, j) n} 2^{(i+j) n}}{\left[u_{1}\left(B_{i}\right)\right]^{1 / q_{1}}\left[u_{2}\left(B_{j}\right)\right]^{1 / q_{2}}}\left\|f \chi_{i}\right\|_{q_{1}, u_{1}}\left\|g \chi_{j}\right\|_{q_{2}, u_{2}}
\end{aligned}
$$


Observing $\left[u\left(B_{k}\right)\right]^{1 / q} \leq\left[u_{1}\left(B_{k}\right)\right]^{1 / q_{1}}\left[u_{2}\left(B_{k}\right)\right]^{1 / q_{2}}$, so we get

$$
\begin{aligned}
& v\left(B_{k}\right)^{\alpha / n}\left\|\mathcal{T}\left(f \chi_{i}, g \chi_{j}\right) \chi_{k}\right\|_{q, u} \\
\leq & C 2^{-\max (k, i) n} 2^{i n}\left[\frac{v\left(B_{k}\right)}{v\left(B_{i}\right)}\right]^{\alpha_{1} / n}\left[\frac{u_{1}\left(B_{k}\right)}{u_{1}\left(B_{i}\right)}\right]^{1 / q_{1}}\left[v\left(B_{i}\right)\right]^{\alpha_{1} / n}\left\|f \chi_{i}\right\|_{q_{1}, u_{1}} \\
& \times 2^{-\max (k, j) n} 2^{j n}\left[\frac{v\left(B_{k}\right)}{v\left(B_{j}\right)}\right]^{\alpha_{2} / n}\left[\frac{u_{2}\left(B_{k}\right)}{u_{2}\left(B_{j}\right)}\right]^{1 / q_{2}}\left[v\left(B_{j}\right)\right]^{\alpha_{2} / n}\left\|g \chi_{j}\right\|_{q_{2}, u_{2}} .
\end{aligned}
$$

Recall the property (1.3) we can see that

$$
2^{-\max (k, i) n} 2^{i n}\left[\frac{v\left(B_{k}\right)}{v\left(B_{i}\right)}\right]^{\alpha_{1} / n}\left[\frac{u_{1}\left(B_{k}\right)}{u_{1}\left(B_{i}\right)}\right]^{1 / q_{1}} \leq C E_{1}(k, i),
$$

and

$$
2^{-\max (k, j) n} 2^{j n}\left[\frac{v\left(B_{k}\right)}{v\left(B_{j}\right)}\right]^{\alpha_{2} / n}\left[\frac{u_{2}\left(B_{k}\right)}{u_{2}\left(B_{j}\right)}\right]^{1 / q_{2}} \leq C E_{2}(k, j) .
$$

Thus we obtain the lemma.

Now we give the proof of Theorem 1.5. If $q \geq 1$, by the Minkowski inequality and Lemma 2.1, we have

$$
\begin{aligned}
& v\left(B_{k}\right)^{\alpha / n}\left\|\mathcal{T}(f, g) \chi_{k}\right\|_{q, u}=v\left(B_{k}\right)^{\alpha / n}\left\|\sum_{i=-\infty}^{\infty} \sum_{j=-\infty}^{\infty} \mathcal{T}\left(f \chi_{i}, g \chi_{j}\right) \chi_{k}\right\|_{q, u} \\
\leq & \sum_{i=-\infty}^{\infty} \sum_{j=-\infty}^{\infty} v\left(B_{k}\right)^{\alpha / n}\left\|\mathcal{T}\left(f \chi_{i}, g \chi_{j}\right) \chi_{k}\right\|_{q, u} \\
\leq & C \sum_{i=-\infty}^{\infty} E_{1}(k, i) v\left(B_{i}\right)^{\alpha_{1} / n}\left\|f \chi_{i}\right\|_{q_{1}, u_{1}} \sum_{j=-\infty}^{\infty} E_{2}(k, j) v\left(B_{j}\right)^{\alpha_{2} / n}\left\|g \chi_{j}\right\|_{q_{2}, u_{2}} \\
\leq & C \sum_{i=-\infty}^{\infty} E_{1}(k, i)^{1-\varepsilon} v\left(B_{i}\right)^{\alpha_{1} / n}\left\|f \chi_{i}\right\|_{q_{1}, u_{1}} \sum_{j=-\infty}^{\infty} E_{2}(k, j)^{1-\varepsilon} v\left(B_{j}\right)^{\alpha_{2} / n}\left\|g \chi_{j}\right\|_{q_{2}, u_{2}}
\end{aligned}
$$

for any $0<\varepsilon<1$, since $E_{1}(k, i)+E_{2}(k, j) \rightarrow 0$ whenever $i, j \rightarrow \pm \infty$.

If $0<q<1$, by the inequality $\left(\sum\left|a_{i}\right|\right)^{q} \leq \sum\left|a_{i}\right|^{q}$ and Lemma 2.1 we have

$$
\begin{aligned}
& v\left(B_{k}\right)^{\alpha / n}\left\|\mathcal{T}(f, g) \chi_{k}\right\|_{q, u} \\
= & v\left(B_{k}\right)^{\alpha / n}\left\{\int_{E_{k}}\left|\sum_{i=-\infty}^{\infty} \sum_{j=-\infty}^{\infty} \mathcal{T}\left(f \chi_{i}, g \chi_{j}\right)(x)\right|^{q} u(x)^{q} d x\right\}^{1 / q}
\end{aligned}
$$




$$
\begin{aligned}
& \leq v\left(B_{k}\right)^{\alpha / n}\left\{\sum_{i=-\infty}^{\infty} \sum_{j=-\infty}^{\infty} \int_{E_{k}}\left|\mathcal{T}\left(f \chi_{i}, g \chi_{j}\right)(x)\right|^{q} u(x)^{q} d x\right\}^{1 / q} \\
& \leq\left\{\sum_{i=-\infty}^{\infty} \sum_{j=-\infty}^{\infty}\left[v\left(B_{k}\right)^{\alpha / n}\left\|\mathcal{T}\left(f \chi_{i}, g \chi_{j}\right) \chi_{k}\right\|_{q, u}\right]^{q}\right\}^{1 / q} \\
& \leq\left\{\sum_{i=-\infty}^{\infty} E_{1}(k, i)^{q} v\left(B_{i}\right)^{\alpha_{1} q / n}\left\|f \chi_{i}\right\|_{q_{1}, u_{1}}^{q} \sum_{j=-\infty}^{\infty} E_{2}(k, j)^{q} v\left(B_{j}\right)^{\alpha_{2} q / n}\left\|g \chi_{j}\right\|_{q_{2}, u_{2}}^{q}\right\}^{1 / q} \\
& \leq C \sum_{i=-\infty}^{\infty} E_{1}(k, i)^{1-\varepsilon} v\left(B_{i}\right)^{\alpha_{1} q / n}\left\|f \chi_{i}\right\|_{q_{1}, u_{1}}^{q}\left\{\sum_{i=-\infty}^{\infty}\left[E_{1}(k, i)\right]^{\frac{\varepsilon}{(1-q)}}\right\}^{\frac{(1-q)}{q}} \\
& \quad \times \sum_{j=-\infty}^{\infty} E_{2}(k, j)^{1-\varepsilon} v\left(B_{j}\right)^{\alpha_{2} q / n}\left\|g \chi_{j}\right\|_{q_{2}, u_{2}}^{q}\left\{\sum_{j=-\infty}^{\infty}\left[E_{2}(k, j)\right]^{\frac{\varepsilon}{(1-q)}}\right\}^{\frac{(1-q)}{q}} \\
& \leq C \sum_{i=-\infty}^{\infty} E_{1}(k, i)^{1-\varepsilon} v\left(B_{i}\right)^{\alpha_{1} q / n}\left\|f \chi_{i}\right\|_{q_{1}, u_{1}}^{q} \sum_{j=-\infty}^{\infty} E_{2}(k, j)^{1-\varepsilon} v\left(B_{j}\right)^{\alpha_{2} q / n}\left\|g \chi_{j}\right\|_{q_{2}, u_{2}}^{q}
\end{aligned}
$$

for any $0<\varepsilon<1$, where in the last inequality we have used the fact that

$$
\sum_{i=-\infty}^{\infty}\left[E_{1}(k, i)\right]^{\gamma}+\sum_{j=-\infty}^{\infty}\left[E_{2}(k, j)\right]^{\gamma}<\infty \text { for any } \gamma>0
$$

because of the condition $\alpha_{\tau} q_{v}<n\left(1-\frac{q_{u_{\tau}}}{q_{\tau}}\right)$ and $\alpha_{\tau} \delta_{v}+\frac{n \delta_{u_{\tau}}}{q_{\tau}}>\lambda_{\tau} q_{v}>0$ for $\tau=1,2$.

Therefore, for any $q>0$ and any $0<\varepsilon<1$ we have that

$$
\begin{aligned}
& \|\mathcal{T}(f, g)\|_{M \dot{K}_{p, q}^{\alpha, \lambda}(v, u)}=\sup _{k_{0} \in Z} v\left(B_{k_{0}}\right)^{\frac{-\lambda}{n}}\left\{\sum_{k=-\infty}^{k_{0}} v\left(B_{k}\right)^{\frac{\alpha p}{n}}\left\|\mathcal{T}(f, g) \chi_{k}\right\|_{q, u}^{p}\right\}^{\frac{1}{p}} \\
& \leq C \sup _{k_{0} \in Z} v\left(B_{k_{0}}\right)^{\frac{-\lambda}{n}}\left\{\sum_{k=-\infty}^{k_{0}}\left\{\sum_{i=-\infty}^{\infty} E_{1}(k, i)^{1-\varepsilon} v\left(B_{i}\right)^{\alpha_{1} / n}\left\|f \chi_{i}\right\|_{q_{1}, u_{1}}\right\}^{p}\right. \\
& \left.\times\left\{\sum_{j=-\infty}^{\infty} E_{2}(k, j)^{1-\varepsilon} v\left(B_{j}\right)^{\alpha_{2} / n}\left\|g \chi_{j}\right\|_{q_{2}, u_{2}}\right\}^{\frac{1}{p}}\right\}^{\alpha^{2}} \\
& \leq C \sup _{k_{0} \in Z} v\left(B_{k_{0}}\right)^{\frac{-\lambda}{n}}\left\{\sum_{k=-\infty}^{k_{0}}\left\{\sum_{i=-\infty}^{\infty} E_{1}(k, i)^{1-\varepsilon} v\left(B_{i}\right)^{\alpha_{1} / n}\left\|f \chi_{i}\right\|_{q_{1}, u_{1}}\right\}^{p_{1}}\right\}^{\frac{1}{p_{1}}}
\end{aligned}
$$




$$
\begin{aligned}
& \times\left\{\sum_{k=-\infty}^{k_{0}}\left\{\sum_{j=-\infty}^{\infty} E_{2}(k, j)^{1-\varepsilon} v\left(B_{j}\right)^{\alpha_{2} / n}\left\|g \chi_{j}\right\|_{q_{2}, u_{2}}\right\}^{p_{2}}\right\}^{\frac{1}{p_{2}}} \\
\leq & C \sup _{k_{0} \in Z} v\left(B_{k_{0}}\right)^{\frac{-\lambda_{1}}{n}}\left\{\sum_{k=-\infty}^{k_{0}}\left\{\sum_{i=-\infty}^{\infty} E_{1}(k, i)^{1-\varepsilon} v\left(B_{i}\right)^{\alpha_{1} / n}\left\|f \chi_{i}\right\|_{q_{1}, u_{1}}\right\}^{p_{1}}\right\}^{\frac{1}{p_{1}}} \\
& \times \sup _{k_{0} \in Z} v\left(B_{k_{0}}\right)^{\frac{-\lambda_{2}}{n}}\left\{\sum_{k=-\infty}^{k_{0}}\left\{\sum_{j=-\infty}^{\infty} E_{2}(k, j)^{1-\varepsilon} v\left(B_{j}\right)^{\alpha_{2} / n}\left\|g \chi_{j}\right\|_{q_{2}, u_{2}}\right\}^{\frac{1}{p_{2}}}\right. \\
:= & J_{1} \times J_{2} .
\end{aligned}
$$

It's enough to show that $J_{1} \leq C\|f\|_{M \dot{K}_{p_{1}, q_{1}}^{\alpha_{1}, \lambda_{1}}\left(v, u_{1}\right)}$ and $J_{2} \leq C\|g\|_{M \dot{K}_{p_{2}, q_{2}}^{\alpha_{2}, \lambda_{2}}\left(v, u_{2}\right)}$. By the symmetry, we only give the estimate for $J_{1}$. We first write that

$$
\begin{aligned}
J_{1} \leq & \sup _{k_{0} \in Z} v\left(B_{k_{0}}\right)^{\frac{-\lambda_{1}}{n}}\left\{\sum_{k=-\infty}^{k_{0}}\left[\sum_{i=-\infty}^{k-2} E_{1}(k, i)^{1-\varepsilon} v\left(B_{i}\right)^{\alpha_{1} / n}\left\|f \chi_{i}\right\|_{q_{1}, u_{1}}\right]^{p_{1}}\right\}^{1 / p_{1}} \\
& +\sup _{k_{0} \in Z} v\left(B_{k_{0}}\right)^{\frac{-\lambda_{1}}{n}}\left\{\sum_{k=-\infty}^{k_{0}}\left[\sum_{i=k-1}^{k+1} E_{1}(k, i)^{1-\varepsilon} v\left(B_{i}\right)^{\alpha_{1} / n}\left\|f \chi_{i}\right\|_{q_{1}, u_{1}}\right]^{p_{1}}\right\}^{1 / p_{1}} \\
& +\sup _{k_{0} \in Z} v\left(B_{k_{0}}\right)^{\frac{-\lambda_{1}}{n}}\left\{\sum_{k=-\infty}^{k_{0}}\left[\sum_{i=k+2}^{\infty} E_{1}(k, i)^{1-\varepsilon} v\left(B_{i}\right)^{\alpha_{1} / n}\left\|f \chi_{i}\right\|_{q_{1}, u_{1}}\right]^{p_{1}}\right\}^{1 / p_{1}} \\
& :=J_{11}+J_{12}+J_{13} .
\end{aligned}
$$

If $0<p_{1}<1$, then by the inequality $\left(\sum\left|a_{i}\right|\right)^{p_{1}} \leq \sum\left|a_{i}\right|^{p_{1}}$ and the inequality (2.1), one can see that

$$
\begin{aligned}
J_{11} & \leq \sup _{k_{0} \in Z} v\left(B_{k_{0}}\right)^{\frac{-\lambda_{1}}{n}}\left\{\sum_{k=-\infty}^{k_{0}}\left[\sum_{i=-\infty}^{k-2} E_{1}(k, i)^{p_{1}(1-\varepsilon)} v\left(B_{i}\right)^{\alpha_{1} p_{1} / n}\left\|f \chi_{i}\right\|_{q_{1}, u_{1}}^{p_{1}}\right]\right\}^{1 / p_{1}} \\
& \leq \sup _{k_{0} \in Z} v\left(B_{k_{0}}\right)^{\frac{-\lambda_{1}}{n}}\left\{\sum_{i=-\infty}^{k_{0}-2}\left[v\left(B_{i}\right)^{\alpha_{1} p_{1} / n}\left\|f \chi_{i}\right\|_{q_{1}, u_{1}}^{p_{1}}\right]\left[\sum_{k=i+2}^{\infty} E_{1}(k, i)^{p_{1}(1-\varepsilon)}\right]\right\}^{1 / p_{1}} \\
& \leq C \sup _{k_{0} \in Z} v\left(B_{k_{0}}\right)^{\frac{-\lambda_{1}}{n}}\left\{\sum_{i=-\infty}^{k_{0}-2}\left[v\left(B_{i}\right)^{\alpha_{1} p_{1} / n}\left\|f \chi_{i}\right\|_{q_{1}, u_{1}}^{p_{1}}\right]\right\}^{1 / p_{1}} \\
& \leq C \sup _{k_{0} \in Z} v\left(B_{k_{0}}\right)^{\frac{-\lambda_{1}}{n}}\left\{\sum_{i=-\infty}^{k_{0}}\left[v\left(B_{i}\right)^{\alpha_{1} p_{1} / n}\left\|f \chi_{i}\right\|_{q_{1}, u_{1}}^{p_{1}}\right]\right\}^{1 / p_{1}} \\
& =C\|f\|_{M \dot{K}_{p_{1}, q_{1}}^{\alpha_{1}, \lambda_{1}}\left(v, u_{1}\right)} .
\end{aligned}
$$

If $p_{1}>1$, the Hölder inequality and the inequality (2.1) yield 


$$
\begin{aligned}
J_{11} \leq & \sup _{k_{0} \in Z} v\left(B_{k_{0}}\right)^{\frac{-\lambda_{1}}{n}}\left\{\sum_{k=-\infty}^{k_{0}}\left[\sum_{i=-\infty}^{k-2} E_{1}(k, i)^{p_{1}(1-\varepsilon)} v\left(B_{i}\right)^{\alpha_{1} p_{1} / n}\left\|f \chi_{i}\right\|_{q_{1}, u_{1}}^{p_{1}}\right]\right. \\
& \left.\times\left[\sum_{i=-\infty}^{k-2} E_{1}(k, i)^{p_{1}^{\prime}(1-\varepsilon)}\right]^{\frac{p_{1}}{p_{1}^{\prime}}}\right\} \\
\leq & C \sup _{k_{0} \in Z} v\left(B_{k_{0}}\right)^{\frac{-\lambda_{1}}{n}}\left\{\sum_{k=-\infty}^{k_{0}} \sum_{i=-\infty}^{k-2} E_{1}(k, i)^{p_{1}(1-\varepsilon)} v\left(B_{i}\right)^{\alpha_{1} p_{1} / n}\left\|f \chi_{i}\right\|_{q_{1}, u_{1}}^{p_{1}}\right\}^{1 / p_{1}} \\
\leq & C \sup _{k_{0} \in Z} v\left(B_{k_{0}}\right)^{\frac{-\lambda_{1}}{n}}\left\{\sum_{i=-\infty}^{k_{0}-2}\left[v\left(B_{i}\right)^{\alpha_{1} p_{1} / n}\left\|f \chi_{i}\right\|_{q_{1}, u_{1}}^{p_{1}}\right]\left[\sum_{k=i+2}^{\infty} E_{1}(k, i)^{p_{1}(1-\varepsilon)}\right]\right\}^{1 / p_{1}} \\
\leq & C \sup _{k_{0} \in Z} v\left(B_{k_{0}}\right)^{\frac{-\lambda_{1}}{n}}\left\{\sum_{i=-\infty}^{k_{0}-2} v\left(B_{i}\right)^{\alpha_{1} p_{1} / n}\left\|f \chi_{i}\right\|_{q_{1}, u_{1}}^{p_{1}}\right\}^{1 / p_{1}} \\
\leq & C\|f\|_{M \dot{K}_{p_{1}, q_{1}}^{\alpha_{1}, \lambda_{1}}\left(v, u_{1}\right)}
\end{aligned}
$$

Thus we have obtained for any $p_{1}>0$ that $J_{11} \leq C\|f\|_{M \dot{K}_{p_{1}, q_{1}}^{\alpha_{1}, \lambda_{1}}\left(v, u_{1}\right)}$.

For $J_{12}$, we have

$$
\begin{aligned}
J_{12} & \leq \sup _{k_{0} \in Z} v\left(B_{k_{0}}\right)^{\frac{-\lambda_{1}}{n}}\left\{\sum_{k=-\infty}^{k_{0}} \sum_{i=k-1}^{k+1} E_{1}(k, i)^{p_{1}(1-\varepsilon)} v\left(B_{i}\right)^{\alpha_{1} p_{1} / n}\left\|f \chi_{i}\right\|_{q_{1}, u_{1}}^{p_{1}}\right\}^{1 / p_{1}} \\
& \leq \sup _{k_{0} \in Z} v\left(B_{k_{0}}\right)^{\frac{-\lambda_{1}}{n}}\left\{\sum_{i=-\infty}^{k_{0}+1} v\left(B_{i}\right)^{\alpha_{1} p_{1} / n}\left\|f \chi_{i}\right\|_{q_{1}, u_{1}}^{p_{1}}\left[\sum_{k=i-1}^{i+1} E_{1}(k, i)^{p_{1}(1-\varepsilon)}\right]\right\}^{1 / p_{1}} \\
& \leq C \sup _{k_{0} \in Z} v\left(B_{k_{0}}\right)^{\frac{-\lambda_{1}}{n}}\left\{\sum_{i=-\infty}^{k_{0}+1} v\left(B_{i}\right)^{\alpha_{1} p_{1} / n}\left\|f \chi_{i}\right\|_{q_{1}, u_{1}}^{p_{1}}\right\}^{1 / p_{1}} \\
& \leq C \sup _{k_{0} \in Z} v\left(B_{k_{0}+1}\right)^{\frac{-\lambda_{1}}{n}}\left\{\sum_{i=-\infty}^{k_{0}+1}\left[v\left(B_{i}\right)^{\alpha_{1} p_{1} / n}\left\|f \chi_{i}\right\|_{q_{1}, u_{1}}^{p_{1}}\right]\right\}^{1 / p_{1}}\left[\frac{v\left(B_{k_{0}}\right)}{v\left(B_{k_{0}+1}\right)}\right]^{\frac{-\lambda_{1}}{n}} \\
& \leq C \sup _{k_{0} \in Z} v\left(B_{k_{0}+1}\right)^{\frac{-\lambda_{1}}{n}}\left\{\sum_{i=-\infty}^{k_{0}+1}\left[v\left(B_{i}\right)^{\alpha_{1} p_{1} / n}\left\|f \chi_{i}\right\|_{q_{1}, u_{1}}^{p_{1}}\right]\right\}^{1 / p_{1}} \\
& =C\|f\|_{M \dot{K}_{p_{1}, q_{1}}^{\alpha_{1}, \lambda_{1}}\left(v, u_{1}\right)} \cdot
\end{aligned}
$$

For $J_{13}$, we decompose it further into two parts as follows

$$
J_{13}=\sup _{k_{0} \in Z} v\left(B_{k_{0}}\right)^{\frac{-\lambda_{1}}{n}}\left\{\sum _ { k = - \infty } ^ { k _ { 0 } } \left[\sum_{i=k+2}^{k_{0}} E_{1}(k, i)^{1-\varepsilon} v\left(B_{i}\right)^{\alpha_{1} / n}\left\|f \chi_{i}\right\|_{q_{1}, u_{1}}\right.\right.
$$




$$
\begin{aligned}
& \left.\left.+\sum_{i=k_{0}+1}^{\infty} E_{1}(k, i)^{1-\varepsilon} v\left(B_{i}\right)^{\alpha_{1} / n}\left\|f \chi_{i}\right\|_{q_{1}, u_{1}}\right]^{p_{1}}\right\}^{1 / p_{1}} \\
\leq & \sup _{k_{0} \in Z} v\left(B_{k_{0}}\right)^{\frac{-\lambda_{1}}{n}}\left\{\sum_{k=-\infty}^{k_{0}}\left[\sum_{i=k+2}^{k_{0}} E_{1}(k, i)^{1-\varepsilon} v\left(B_{i}\right)^{\alpha_{1} / n}\left\|f \chi_{i}\right\|_{q_{1}, u_{1}}\right]^{p_{1}}\right\}^{1 / p_{1}} \\
& +\sup _{k_{0} \in Z} v\left(B_{k_{0}}\right)^{\frac{-\lambda_{1}}{n}}\left\{\sum_{k=-\infty}^{k_{0}}\left[\sum_{i=k_{0}+1}^{\infty} E_{1}(k, i)^{1-\varepsilon} v\left(B_{i}\right)^{\alpha_{1} / n}\left\|f \chi_{i}\right\|_{q_{1}, u_{1}}\right]^{p_{1}}\right\}^{1 / p_{1}} \\
:= & J_{131}+J_{132} .
\end{aligned}
$$

Follow the same way used in the estimation for $J_{11}$, we get $J_{131} \leq C\|f\|_{M \dot{K}_{p_{1}, q_{1}}^{\alpha_{1}, \lambda_{1}}\left(v, u_{1}\right)}$. It's left to estimate the term $J_{132}$. First noting that

$$
\begin{aligned}
& J_{132} \leq \sup _{k_{0} \in Z} v\left(B_{k_{0}}\right)^{\frac{-\lambda_{1}}{n}} \\
& \times\left\{\sum_{k=-\infty}^{k_{0}}\left[\sum_{i=k_{0}+1}^{\infty} E_{1}(k, i)^{1-\varepsilon}\left(v\left(B_{i}\right)^{\alpha_{1} p_{1} / n}\left\|f \chi_{i}\right\|_{q_{1}, u_{1}}^{p_{1}}\right)^{\frac{1}{p_{1}}}\right]^{p_{1}}\right\}^{1 / p_{1}} \\
& \leq \sup _{k_{0} \in Z} v\left(B_{k_{0}}\right)^{\frac{-\lambda_{1}}{n}}\left\{\sum_{k=-\infty}^{k_{0}}\left[\sum_{i=k_{0}+1}^{\infty} E_{1}(k, i)^{1-\varepsilon} v\left(B_{i}\right)^{\frac{\lambda_{1}}{n}}\|f\|_{M \dot{K}_{p_{1}, q_{1}}^{\alpha_{1}, \lambda_{1}}}\left(v, u_{1}\right)\right]^{p_{1}}\right\}^{1 / p_{1}} \\
& \leq\|f\|_{M \dot{K}_{p_{1}, q_{1}}^{\alpha_{1}, \lambda_{1}}\left(v, u_{1}\right)} \sup _{k_{0} \in Z} v\left(B_{k_{0}}\right)^{\frac{-\lambda_{1}}{n}}\left\{\sum_{k=-\infty}^{k_{0}}\left[\sum_{i=k_{0}+1}^{\infty} E_{1}(k, i)^{1-\varepsilon} v\left(B_{i}\right)^{\frac{\lambda_{1}}{n}}\right]^{p_{1}}\right\}^{1 / p_{1}} \\
& \leq C\|f\|_{M \dot{K}_{p_{1}, q_{1}}^{\alpha_{1}, \lambda_{1}}\left(v, u_{1}\right)} \sup _{k_{0} \in Z}\left\{\sum_{k=-\infty}^{k_{0}}\left[\sum_{i=k_{0}+1}^{\infty} E_{1}(k, i)^{1-\varepsilon}\left(\frac{v\left(B_{i}\right)}{v\left(B_{k_{0}}\right)}\right)^{\frac{\lambda_{1}}{n}}\right]^{p_{1}}\right\}^{1 / p_{1}} \\
& \leq C\|f\|_{M \dot{K}_{p_{1}, q_{1}}^{\alpha_{1}, \lambda_{1}}\left(v, u_{1}\right)} \sup _{k_{0} \in Z}\left\{\sum_{k=-\infty}^{k_{0}}\left[\sum_{i=k_{0}+1}^{\infty} E_{1}(k, i)^{1-\varepsilon_{2}} 2^{\left(i-k_{0}\right) \lambda_{1} q_{v}}\right]^{p_{1}}\right\}^{1 / p_{1}} .
\end{aligned}
$$

We remark that the condition $\alpha_{i} \delta_{v}>\frac{-n \delta_{u_{i}}}{q_{i}}+\lambda_{i} q_{v}$ implies thet $\left(\alpha_{i} \delta_{v}+\frac{n \delta_{u_{i}}}{q_{i}}\right)(1-$ $\varepsilon)-\lambda_{i} q_{v}>0$ for some small enough positive number $\varepsilon$. Hence, from the definition of $E_{1}(k, i)$ we see that

$$
\sum_{k=-\infty}^{k_{0}}\left[\sum_{i=k_{0}+1}^{\infty} E_{1}(k, i)^{1-\varepsilon} 2^{\left(i-k_{0}\right) \lambda_{1} q_{v}}\right]^{p_{1}}
$$




$$
\begin{aligned}
& =\sum_{k=-\infty}^{k_{0}}\left[2^{k(1-\varepsilon)\left(\alpha_{1} \delta_{v}+n \delta_{u_{1}} / q_{1}\right)-k_{0} \lambda_{1} q_{v}} \sum_{i=k_{0}+1}^{\infty} 2^{-i(1-\varepsilon)\left(\alpha_{1} \delta_{v}+n \delta_{u_{1}} / q_{1}\right)+i \lambda_{1} q_{v}}\right]^{p_{1}} \\
& \leq C \sum_{k=-\infty}^{k_{0}}\left[2^{\left(k-k_{0}\right)(1-\varepsilon)\left(\alpha_{1} \delta_{v}+n \delta_{u_{1}} / q_{1}\right)}\right]^{p_{1}} \leq C
\end{aligned}
$$

with the positive constant $C$ independent of $k_{0}$. Thus we obtain

$$
J_{132} \leq C\|f\|_{M \dot{K}_{p_{1}, q_{1}}^{\alpha_{1}, \lambda_{1}}\left(v, u_{1}\right)} .
$$

Now combining all the estimates above we reach at

$$
J_{1} \leq J_{11}+J_{12}+J_{131}+J_{132} \leq C\|f\|_{M \dot{K}_{p_{1}, q_{1}}^{\alpha_{1}, \lambda_{1}}\left(v, u_{1}\right)} .
$$

By symmetry, we have $J_{2} \leq C\|g\|_{M \dot{K}_{p_{2}, q_{2}}^{\alpha_{2}, \lambda_{2}}\left(v, u_{2}\right)}$. Then we get

$$
\|\mathcal{T}(f, g)\|_{M \dot{K}_{p, q}^{\alpha, \lambda}(v, u)} \leq C\|f\|_{M \dot{K}_{p_{1}, q_{1}}^{\alpha_{1}, \lambda_{1}}\left(v, u_{1}\right)}\|g\|_{M \dot{K}_{p_{2}, q_{2}}^{\alpha_{2}, \lambda_{2}}\left(v, u_{2}\right)} .
$$

The proof of Theorem 1.5 is complete.

\section{The Proof of Theorem 1.6 And 1.7}

We first give the proof of Theorem 1.6. Let $\Lambda_{1}=\{(i, j): k-1 \leq i, j \leq k$ $+1\}$ and $\Lambda_{2}=\mathbb{Z}^{2} \backslash \Lambda_{1}$, then

$$
\begin{aligned}
|\mathcal{T}(f, g)(x)| & \leq\left|\mathcal{T}\left(\sum_{i=k-1}^{k+1} f \chi_{i}, \sum_{j=k-1}^{k+1} g \chi_{j}\right)(x)\right|+\sum_{i, j \in \Lambda_{2}}\left|\mathcal{T}\left(f \chi_{i}, g \chi_{j}\right)(x)\right| \\
& :=L_{1}(x)+L_{2}(x) .
\end{aligned}
$$

We can write the following decomposition

$$
\begin{aligned}
& \|\mathcal{T}(f, g)\|_{W M \dot{K}_{p, q}^{\alpha, \lambda}(v, u)} \\
& \leq \sup _{k_{0} \in Z} v\left(B_{k_{0}}\right)^{\frac{-\lambda}{n}} \sup _{t>0} t\left\{\sum_{k=-\infty}^{k_{0}} v\left(B_{k}\right)^{\frac{\alpha p}{n}}\left[u\left(\left\{x \in E_{k}:|\mathcal{T}(f, g)(x)|>t\right\}\right)\right]^{\frac{p}{q}}\right\}^{\frac{1}{p}} \\
& \leq \sup _{k_{0} \in Z} v\left(B_{k_{0}}\right)^{\frac{-\lambda}{n}} \sup _{t>0} t\left\{\sum_{k=-\infty}^{k_{0}} v\left(B_{k}\right)^{\frac{\alpha p}{n}}\left[u\left(\left\{x \in E_{k}:\left|L_{1}(x)\right|>t / 2\right\}\right)\right]^{\frac{p}{q}}\right\}^{\frac{1}{p}} \\
& \quad+\sup _{k_{0} \in Z} v\left(B_{k_{0}}\right)^{\frac{-\lambda}{n}} \sup _{t>0} t\left\{\sum_{k=-\infty}^{k_{0}} v\left(B_{k}\right)^{\frac{\alpha p}{n}}\left[u\left(\left\{x \in E_{k}:\left|L_{2}(x)\right|>t / 2\right\}\right)\right]^{\frac{p}{q}}\right\}^{\frac{1}{p}} \\
& :=H_{1}+H_{2} .
\end{aligned}
$$


By the weak boundedness of $\mathcal{T}$ and the Hölder inequality, and using the same method as for $J_{12}$, we get

$$
\begin{aligned}
H_{1} \leq & C \sup _{k_{0} \in Z} v\left(B_{k_{0}}\right)^{\frac{-\lambda}{n}}\left\{\sum_{k=-\infty}^{k_{0}} v\left(B_{k}\right)^{\frac{\alpha p}{n}}\left(\sum_{i=k-1}^{k+1}\left\|f \chi_{i}\right\|_{q_{1}, u_{1}} \cdot \sum_{j=k-1}^{k+1}\left\|g \chi_{j}\right\|_{q_{2}, u_{2}}\right)^{p}\right\}^{\frac{1}{p}} \\
\leq & C \sup _{k_{0} \in Z} v\left(B_{k_{0}}\right)^{\frac{-\lambda_{1}}{n}}\left\{\sum_{k=-\infty}^{k_{0}} \sum_{i=k-1}^{k+1} v\left(B_{i}\right)^{\frac{\alpha_{1} p_{1}}{n}}\left\|f \chi_{i}\right\|_{q_{1}, u_{1}}^{p_{1}}\left[\frac{v\left(B_{k}\right)}{v\left(B_{i}\right)}\right]^{\frac{\alpha_{1} p_{1}}{n}}\right\}^{\frac{1}{p_{1}}} \\
& \times \sup _{k_{0} \in Z} v\left(B_{k_{0}}\right)^{\frac{-\lambda_{2}}{n}}\left\{\sum_{k=-\infty}^{k_{0}} \sum_{j=k-1}^{k+1} v\left(B_{j}\right)^{\frac{\alpha_{2} p_{2}}{n}}\left\|g \chi_{j}\right\|_{q_{2}, u_{2}}^{p_{2}}\left[\frac{v\left(B_{k}\right)}{v\left(B_{j}\right)}\right]^{\frac{\alpha_{2} p_{2}}{n}}\right\}^{\frac{1}{p_{2}}} \\
\leq & C \sup _{k_{0} \in Z} v\left(B_{k_{0}}\right)^{\frac{-\lambda_{1}}{n}}\left\{\sum_{i=-\infty}^{k_{0}+1} v\left(B_{i}\right)^{\frac{\alpha_{1} p_{1}}{n}}\left\|f \chi_{i}\right\|_{q_{1}, u_{1}}^{p_{1}} \sum_{k=i-1}^{i+1}\left[\frac{v\left(B_{k}\right)}{v\left(B_{i}\right)}\right]^{\frac{\alpha_{1} p_{1}}{n}}\right\}^{\frac{1}{p_{1}}} \\
& \times \sup _{k_{0} \in Z} v\left(B_{k_{0}}\right)^{\frac{-\lambda_{2}}{n}}\left\{\sum_{j=-\infty}^{k_{0}+1} v\left(B_{j}\right)^{\frac{\alpha_{2} p_{2}}{n}}\left\|g \chi_{j}\right\|_{q_{2}, u_{2}}^{p_{2}} \sum_{k=j-1}^{j+1}\left[\frac{v\left(B_{k}\right)}{v\left(B_{j}\right)}\right]^{\frac{\alpha_{2} p_{2}}{n}}\right\}^{\frac{1}{p_{2}}} \\
\leq & C\|f\|_{M \dot{K}_{p_{1}, q_{1}}^{\alpha_{1}, \lambda_{1}}\left(v, u_{1}\right)}\|g\|_{M \dot{K}_{p_{2}, q_{2}}^{\alpha_{2}, \lambda_{2}}\left(v, u_{2}\right)}
\end{aligned}
$$

As for $\mathrm{H}_{2}$, It is easy to verify that the estimates in Lemma 2.1 still hold for $(i, j) \in \Lambda_{2}$ even if $q_{1}$ or $q_{2}$ equals to 1 . Thus we have

$$
\begin{aligned}
& t v\left(B_{k}\right)^{\alpha / n} u\left(\left\{x \in E_{k}:\left|L_{1}(x)\right|>t / 2\right\}\right)^{\frac{1}{q}} \leq C v\left(B_{k}\right)^{\alpha / n}\left\|\sum_{(i, j) \in \Lambda_{2}} \mathcal{T}\left(f \chi_{i}, g \chi_{j}\right) \chi_{k}\right\|_{q, u} \\
& \leq C \sum_{(i, j) \in \Lambda_{2}} E_{1}(k, i) v\left(B_{i}\right)^{\alpha_{1} / n}\left\|f \chi_{i}\right\|_{q_{1}, u_{1}} \times E_{2}(k, j) v\left(B_{j}\right)^{\alpha_{2} / n}\left\|g \chi_{j}\right\|_{q_{2}, u_{2}}
\end{aligned}
$$

and so

$$
\begin{aligned}
& H_{2} \leq C \sup _{k_{0} \in Z} v\left(B_{k_{0}}\right)^{\frac{-\lambda_{1}}{n}}\left\{\sum_{k=-\infty}^{k_{0}}\left(\sum_{i \leq k-2, i \geq k+2} E_{1}(k, i) v\left(B_{i}\right)^{\frac{\alpha_{1}}{n}}\left\|f \chi_{i}\right\|_{q_{1}, u_{1}}\right)^{p_{1}}\right\}^{\frac{1}{p_{1}}} \\
& \times \sup _{k_{0} \in Z} v\left(B_{k_{0}}\right)^{\frac{-\lambda_{2}}{n}}\left\{\sum_{k=-\infty}^{k_{0}}\left(\sum_{j \leq k-2, j \geq k+2} E_{2}(k, j) v\left(B_{j}\right)^{\frac{\alpha_{2}}{n}}\left\|g \chi_{i}\right\|_{q_{2}, u_{2}}\right)^{p_{2}}\right\}^{\frac{1}{p_{2}}} \\
& :=I_{1} \cdot I_{2} \text {. }
\end{aligned}
$$

Further, one has 


$$
\begin{aligned}
I_{1} \leq & C \sup _{k_{0} \in Z} v\left(B_{k_{0}}\right)^{\frac{-\lambda_{1}}{n}}\left\{\sum_{k=-\infty}^{k_{0}}\left(\sum_{i=-\infty}^{k-2} E_{1}(k, i) v\left(B_{i}\right)^{\frac{\alpha_{1}}{n}}\left\|f \chi_{i}\right\|_{q_{1}, u_{1}}\right)^{p_{1}}\right\}^{\frac{1}{p_{1}}} \\
& +C \sup _{k_{0} \in Z} v\left(B_{k_{0}}\right)^{\frac{-\lambda_{1}}{n}}\left\{\sum_{k=-\infty}^{k_{0}}\left(\sum_{i=k+2}^{\infty} E_{1}(k, i) v\left(B_{i}\right)^{\frac{\alpha_{1}}{n}}\left\|f \chi_{i}\right\|_{q_{1}, u_{1}}\right)^{p_{1}}\right\}^{\frac{1}{p_{1}}} \\
:= & I_{11}+I_{12} .
\end{aligned}
$$

Now we use the same method as for $J_{11}$ to estimate $I_{11}$, and use the same method as for $J_{13}$ to estimate $I_{12}$. Then we can deduce that

$$
I_{1} \leq C\|f\|_{M \dot{K}_{p_{1}, q_{1}}^{\alpha_{1}, \lambda_{1}}\left(v, u_{1}\right)} .
$$

By symmetry, we have

$$
I_{2} \leq C\|g\|_{M \dot{K}_{p_{2}, q_{2}}^{\alpha_{2}, \lambda_{2}}\left(v, u_{2}\right)} .
$$

Hence

$$
H_{2} \leq C\|f\|_{M \dot{K}_{p_{1}, q_{1}}^{\alpha_{1}, \lambda_{1}}\left(v, u_{1}\right)}\|g\|_{M \dot{K}_{p_{2}, q_{2}}^{\alpha_{2}, \lambda_{2}}\left(v, u_{2}\right)} .
$$

Together with the estimate of $H_{1}$, we finish the proof of Theorem 1.6.

Finally we turn to the proof of Theorem 1.7. Using the same notions defined in the proof of Theorem 1.6. We have the same estimates for $H_{1}$ as above, and we need only give the estimates for $\mathrm{H}_{2}$ under the current hypotheses. Without loss of generality, let $\alpha_{1} q_{v}=n\left(1-q_{u_{1}} / q_{1}\right)$ and $\alpha_{2} q_{v}<n\left(1-q_{u_{2}} / q_{2}\right)$, and $\alpha_{\tau} \delta_{v}>-n \delta_{u_{\tau}} / q_{\tau}+\lambda_{\tau} \delta_{v}$ for $\tau=1,2$.

When $(i, j) \in \Lambda_{2}$, by the estimates in the proof of Lemma 2.1, we obtain

$$
\begin{aligned}
& \quad\left|L_{2}(x) \chi_{k}(x)\right| \leq C \sum_{(i, j) \in \Lambda_{2}} 2^{-2 \max (k, i, j) n} 2^{(i+j) n} \frac{\left\|f \chi_{i}\right\|_{q_{1}, u_{1}}\left\|g \chi_{j}\right\|_{q_{2}, u_{2}}}{\left[u_{1}\left(B_{i}\right)\right]^{1 / q_{1}}\left[u_{2}\left(B_{j}\right)\right]^{1 / q_{2}}} \\
& \leq C \sum_{(i, j) \in \Lambda_{2}} \frac{2^{-2 \max (k, i, j) n} 2^{(i+j) n}}{\left\{\left[u_{1}\left(B_{k}\right)\right]^{1 / q_{1}}\left[u_{2}\left(B_{k}\right)\right]^{1 / q_{2}}\left[v\left(B_{k}\right)\right]^{(\alpha-\lambda) / n}\right\}} \\
& \quad \times\left\{\left[\frac{v\left(B_{k}\right)}{v\left(B_{i}\right)}\right]^{\left(\alpha_{1}-\lambda_{1}\right) / n}\left[\frac{u_{1}\left(B_{k}\right)}{u_{1}\left(B_{i}\right)}\right]^{1 / q_{1}} v\left(B_{i}\right)^{\left(\alpha_{1}-\lambda_{1}\right) / n}\left\|f \chi_{i}\right\|_{q_{1}, u_{1}}\right\} \\
& \leq \frac{\times\left\{\left[\frac{v\left(B_{k}\right)}{v\left(B_{j}\right)}\right]^{\left(\alpha_{2}-\lambda_{2}\right) / n}\left[\frac{u_{2}\left(B_{k}\right)}{u_{2}\left(B_{j}\right)}\right]^{1 / q_{2}} v\left(B_{j}\right)^{\left(\alpha_{2}-\lambda_{2}\right) / n}\left\|g \chi_{j}\right\|_{q_{2}, u_{2}}\right\}}{\left\{\left[u_{1}\left(B_{k}\right)\right]^{\left.1 / q_{1}\left[u_{2}\left(B_{k}\right)\right]^{1 / q_{2}}\left[v\left(B_{k}\right)\right]^{(\alpha-\lambda) / n}\right\}}\right.}
\end{aligned}
$$




$$
\begin{aligned}
& \times\left\{\sum_{i=-\infty}^{k-2} E_{1}(k, i) 2^{-(k-i) \lambda_{1} q_{v}} v\left(B_{i}\right)^{\left(\alpha_{1}-\lambda_{1}\right) / n}\left\|f \chi_{i}\right\|_{q_{1}, u_{1}}\right. \\
& \left.\quad+\sum_{i=k+2}^{\infty} E_{1}(k, i) 2^{-(k-i) \lambda_{1} \delta_{v}} v\left(B_{i}\right)^{\left(\alpha_{1}-\lambda_{1}\right) / n}\left\|f \chi_{i}\right\|_{q_{1}, u_{1}}\right\} \\
& \times\left\{\sum_{j=-\infty}^{k-2} E_{2}(k, j) 2^{-(k-j) \lambda_{2} q_{v}} v\left(B_{j}\right)^{\left(\alpha_{2}-\lambda_{2}\right) / n}\left\|g \chi_{j}\right\|_{q_{2}, u_{2}}\right. \\
& \left.\quad+\sum_{j=k+2}^{\infty} E_{2}(k, j) 2^{-(k-j) \lambda_{2} \delta_{v}} v\left(B_{j}\right)^{\left(\alpha_{2}-\lambda_{2}\right) / n}\left\|g \chi_{j}\right\|_{q_{2}, u_{2}}\right\} .
\end{aligned}
$$

Since $\alpha_{1} q_{v}=n\left(1-q_{u_{1}} / q_{1}\right)$, one has $E_{1}(k, i)=1$ for $i \leq k-2$, and so

$$
\begin{aligned}
& \sum_{i=-\infty}^{k-2} E_{1}(k, i) 2^{-(k-i) \lambda_{1} q_{v}} v\left(B_{i}\right)^{\left(\alpha_{1}-\lambda_{1}\right) / n}\left\|f \chi_{i}\right\|_{q_{1}, u_{1}} \\
& = \begin{cases}\sum_{i=-\infty}^{k-2} v\left(B_{i}\right)^{\alpha_{1} / n}\left\|f \chi_{i}\right\|_{q_{1}, u_{1}}, & \text { if } \lambda_{1}=0 \\
\sum_{i=-\infty} 2^{-(k-i) \lambda_{1} q_{v}} v\left(B_{i}\right)^{-\lambda_{1} / n} v\left(B_{i}\right)^{\alpha_{1} / n}\left\|f \chi_{i}\right\|_{q_{1}, u_{1}}, & \text { if } \lambda_{1}>0\end{cases} \\
& \leq \begin{cases}\|f\|_{M \dot{K}_{p_{1}, q_{1}}^{\alpha_{1}, 0}\left(v, u_{1}\right)}, & \text { if } \lambda_{1}=0 \\
\|f\|_{M \dot{K}_{p_{1}, q_{1}}^{\alpha_{1}, \lambda_{1}}\left(v, u_{1}\right)} \sum_{i=-\infty}^{k-2} 2^{-(k-i) \lambda_{1} q_{v},}, & \text { if } \lambda_{1}>0\end{cases} \\
& \leq C\|f\|_{M \dot{K}_{p_{1}, q_{1}}^{\alpha_{1}, \lambda_{1}}\left(v, u_{1}\right)} .
\end{aligned}
$$

On the other hand, notice the condition $\alpha_{1} \delta_{v}>\frac{-n \delta_{u_{1}}}{q_{1}}+\lambda_{1} \delta_{v}$, we have

$$
\begin{aligned}
& \sum_{i=k+2}^{\infty} E_{1}(k, i) 2^{-(k-i) \lambda_{1} \delta_{v}} v\left(B_{i}\right)^{\left(\alpha_{1}-\lambda_{1}\right) / n}\left\|f \chi_{i}\right\|_{q_{1}, u_{1}} \\
\leq & \|f\|_{M \dot{K}_{p_{1}, q_{1}}^{\alpha_{1}, \lambda_{1}}\left(v, u_{1}\right)} \sum_{i=k+2}^{\infty} E_{1}(k, i) 2^{-(k-i) \lambda_{1} \delta_{v}} \leq C\|f\|_{M \dot{K}_{p_{1}, q_{1}}^{\alpha_{1}, \lambda_{1}}\left(v, u_{1}\right)},
\end{aligned}
$$

so we get

$$
\begin{aligned}
& \sum_{i=-\infty}^{k-2} E_{1}(k, i) 2^{-(k-i) \lambda_{1} q_{v}} v\left(B_{i}\right)^{\left(\alpha_{1}-\lambda_{1}\right) / n}\left\|f \chi_{i}\right\|_{q_{1}, u_{1}} \\
+ & \sum_{i=k+2}^{\infty} E_{1}(k, i) 2^{-(k-i) \lambda_{1} \delta_{v}} v\left(B_{i}\right)^{\left(\alpha_{1}-\lambda_{1}\right) / n}\left\|f \chi_{i}\right\|_{q_{1}, u_{1}} \leq C\|f\|_{M \dot{K}_{p_{1}, q_{1}}^{\alpha_{1}, \lambda_{1}}\left(v, u_{1}\right)} .
\end{aligned}
$$


And in the same way we get

$$
\begin{aligned}
& \sum_{j=-\infty}^{k-2} E_{2}(k, j) 2^{-(k-j) \lambda_{2} q_{v}} v\left(B_{j}\right)^{\left(\alpha_{2}-\lambda_{2}\right) / n}\left\|g \chi_{j}\right\|_{q_{2}, u_{2}} \\
& +\sum_{j=k+2}^{\infty} E_{2}(k, j) 2^{-(k-j) \lambda_{2} \delta_{v}} v\left(B_{j}\right)^{\left(\alpha_{2}-\lambda_{2}\right) / n}\left\|g \chi_{j}\right\|_{q_{2}, u_{2}} \leq C\|g\|_{M \dot{K}_{p_{2}, q_{2}}^{\alpha_{2}, \lambda_{2}}\left(v, u_{2}\right)}
\end{aligned}
$$

Hence we have

$$
\begin{aligned}
& \left|L_{2}(x) \chi_{k}(x)\right| \leq \frac{C\|f\|_{M \dot{K}_{p_{1}, q_{1}}^{\alpha_{1}, \lambda_{1}}\left(v, u_{1}\right)}\|g\|_{M \dot{K}_{p_{2}, q_{2}}^{\alpha_{2}, \lambda_{2}}\left(v, u_{2}\right)}}{\left\{\left[u_{1}\left(B_{k}\right)\right]^{1 / q_{1}}\left[u_{2}\left(B_{k}\right)\right]^{1 / q_{2}}\left[v\left(B_{k}\right)\right]^{(\alpha-\lambda) / n}\right\}} \\
& \leq \frac{C\|f\|_{M \dot{K}_{p_{1}, q_{1}}^{\alpha_{1}, \lambda_{1}}\left(v, u_{1}\right)}\|g\|_{M \dot{K}_{p_{2}, q_{2}}^{\alpha_{2}, \lambda_{2}}\left(v, u_{2}\right)}}{\left\{\left[u_{1}\left(B_{0}\right)\right]^{1 / q_{1}}\left[u_{2}\left(B_{0}\right)\right]^{1 / q_{2}}\left[v\left(B_{0}\right)\right]^{(\alpha-\lambda) / n}\right\}} \\
& \quad \times\left\{\left[\frac{u_{1}\left(B_{0}\right)}{u_{1}\left(B_{k}\right)}\right]^{1 / q_{1}}\left[\frac{v\left(B_{0}\right)}{v\left(B_{k}\right)}\right]^{\alpha_{1} / n}\left[\frac{u_{2}\left(B_{0}\right)}{u_{2}\left(B_{k}\right)}\right]^{1 / q_{2}}\left[\frac{v\left(B_{0}\right)}{v\left(B_{k}\right)}\right]^{\alpha_{2} / n}\right\} \\
& \leq \frac{C 2^{-k\left[(\alpha-\lambda) \delta_{v}+\frac{n \delta_{u_{1}}}{q_{1}}+\frac{n \delta_{u_{2}}}{q_{2}}\right]}\|f\|_{M \dot{K}_{p_{1}, q_{1}}^{\alpha_{1}, \lambda_{1}}\left(v, u_{1}\right)}\|g\|_{M \dot{K}_{p_{2}, q_{2}}^{\alpha_{2}, \lambda_{2}}\left(v, u_{2}\right)}}{\left\{\left[u_{1}\left(B_{0}\right)\right]^{1 / q_{1}}\left[u_{2}\left(B_{0}\right)\right]^{1 / q_{2}}\left[v\left(B_{0}\right)\right]^{(\alpha-\lambda) / n}\right\}} \\
& \rightarrow 0
\end{aligned}
$$

whenever $k \rightarrow+\infty$. Now for any given $t>0$, we set

$$
k_{t}=\max \left\{k \in \mathbb{Z}: t / 2<\frac{C\|f\|_{M \dot{K}_{p_{1}, q_{1}}^{\alpha_{1}, \lambda_{1}}\left(v, u_{1}\right)}\|g\|_{M \dot{K}_{p_{2}, q_{2}}^{\alpha_{2}, \lambda_{2}}\left(v, u_{2}\right)}}{\left\{\left[u_{1}\left(B_{k}\right)\right]^{1 / q_{1}}\left[u_{2}\left(B_{k}\right)\right]^{1 / q_{2}}\left[v\left(B_{k}\right)\right]^{(\alpha-\lambda) / n}\right\}}\right\}
$$

Obviously, if $k \geq k_{t}+1$, then the set $\left\{x \in E_{k}:\left|L_{2}(x)\right|>t / 2\right\}$ is empty. Thus

$$
\begin{aligned}
H_{2}=\sup _{k_{0} \leq k_{t}} v\left(B_{k_{0}}\right)^{\frac{-\lambda}{n}} \sup _{t>0} t & \left\{\sum_{k=-\infty}^{k_{0}} v\left(B_{k}\right)^{\frac{\alpha p}{n}}\left[u\left(\left\{x \in E_{k}:\left|L_{2}(x)\right|>t / 2\right\}\right)\right]^{\frac{p}{q}}\right\}^{\frac{1}{p}} \\
\leq C \sup _{k_{0} \leq k_{t}, t>0} v\left(B_{k_{0}}\right)^{\frac{-\lambda}{n}} & \left\{\sum_{k=-\infty}^{k_{0}} v\left(B_{k}\right)^{\frac{\alpha p}{n}}\left[u\left(B_{k}\right)\right]^{\frac{p}{q}}\right\}^{\frac{1}{p}} \\
& \times \frac{\|f\|_{M \dot{K}_{p_{1}, q_{1}}^{\alpha_{1}, \gamma_{1}}\left(v, u_{1}\right)}\|g\|_{M \dot{K}_{p_{2}, q_{2}}^{\alpha_{2}, \lambda_{2}}\left(v, u_{2}\right)}}{\left\{\left[u_{1}\left(B_{k}\right)\right]^{1 / q_{1}}\left[u_{2}\left(B_{k}\right)\right]^{1 / q_{2}}\left[v\left(B_{k}\right)\right]^{(\alpha-\lambda) / n}\right\}}
\end{aligned}
$$




$$
\begin{aligned}
& \leq C \sup _{k_{0} \leq k_{t}, t>0} \frac{\|f\|_{M \dot{K}_{p_{1}, q_{1}}^{\alpha_{1}, \lambda_{1}}\left(v, u_{1}\right)}\|g\|_{M \dot{K}_{p_{2}, q_{2}}^{\alpha_{2}, \lambda_{2}}\left(v, u_{2}\right)}}{\left\{\left[u_{1}\left(B_{k}\right)\right]^{1 / q_{1}}\left[u_{2}\left(B_{k}\right)\right]^{1 / q_{2}}\left[v\left(B_{k}\right)\right]^{(\alpha-\lambda) / n}\right\}} \\
& \times\left\{\sum_{k=-\infty}^{k_{0}} v\left(B_{k_{0}}\right)^{\frac{-\lambda p}{n}} v\left(B_{k}\right)^{\frac{\alpha p}{n}}\left[u\left(B_{k}\right)\right]^{\frac{p}{q}}\right\}^{\frac{1}{p}} \\
& \leq C\|f\|_{M \dot{K}_{p_{1}, q_{1}}^{\alpha_{1}, \lambda_{1}}\left(v, u_{1}\right)}\|g\|_{M \dot{K}_{p_{2}, q_{2}}^{\alpha_{2}, \lambda_{2}}\left(v, u_{2}\right)} \sup _{k_{0} \leq k_{t}, t>0}\left\{\sum_{k=-\infty}^{k_{0}}\left[\frac{v\left(B_{k_{0}}\right)}{v\left(B_{k}\right)}\right]^{\frac{-\lambda p}{n}}\right\}^{\frac{1}{p}} \\
& \leq C\|f\|_{M \dot{K}_{p_{1}, q_{1}}^{\alpha_{1}, \lambda_{1}}\left(v, u_{1}\right)}\|g\|_{M \dot{K}_{p_{2}, q_{2}}^{\alpha_{2}, \lambda_{2}}\left(v, u_{2}\right)}
\end{aligned}
$$

Combining the estimates for $H_{1}$ and $H_{2}$, we finish the proof of Theorem 1.7.

\section{REFERENCES}

1. A. Beurling, Construction and analysis of some convolution algebras, Ann. Inst. Fourier Grenoble, 14 (1964), 1-32.

2. L. Grafakos and R. Torres, Multilinear Calderon-Zygmund theory, Adv. Math., 165 (2002), 124-164.

3. L. Grafakos and R. Torres, Maximal operator and weighted norm inequalities for multilinear singular interals, Indiana U. Math. J., 51(5) (2002), 1261-1276.

4. C. Herz, Lipschilz spaces and Bernstein's theorem on absoluted convergent Fourier transforms, Math. Mech. J., 18 (1968), 283-324.

5. S.-Z. Lu and D.-C. Yang, The decomposition of weighted Herz spaces on $\mathbb{R}^{n}$ and its application, Sci. in China, Ser. A, 38 (1995), 147-158.

6. Y.-L. Shi and X.-X. Tao, Boundedness for multilinear fractional integral operators on Herz type spaces, Appl. Math., A J. Chinese Univ. Ser. B., 23(4) (2008), 437-446.

7. Y.-L. Shi and X.-X. Tao, Multilinear Riesz potential operators on Herz-type spaces and generalized Morrey spaces, Hokkaido Math. J., 38 (2009), 635-662.

8. E. M. Stein, Harmonic Analysis: Real-Variable Methods, Orthogonality, and Oscillatory Integrals, Princeton Univ. Press, Princeton, NJ, 1993.

9. G.-P. Tao, Boundedness of multilinear operators on weighted Herz type spaces, Anal. Theory Appl., 21(1) (2005), 73-89.

10. X.-X. Tao, Y.-L. Shi and S.-Y. Zhang, Boundedness of multilinear Riesz potential operators on product of Morrey spaces and Herz-Morrey spaces, Acta Math. Sinica, Chinese Ser., 52(3) (2009), 535-548.

11. Q. Wu, Weighted estimates for multilinear Calderon-Zygmund operators, Adv. Math. (in China), 33 (2004), 333-342. 


\author{
Xiangxing Tao \\ Department of Mathematics \\ Zhejiang University of Science and Technology \\ Hangzhou 310023 \\ P. R. China \\ E-mail: xxtao@hotmail.com \\ Huihui Zhang \\ Department of Mathematics \\ Ningbo University \\ Ningbo 315211 \\ P. R. China \\ E-mail: zhanghuihuinb@163.com
}


